\title{
Integrated metabolomics and transcriptome analysis on flavonoid biosynthesis in safflower (Carthamus tinctorius L.) under MeJA treatment
}

Jiang Chen ${ }^{1,2}$, Jie Wang ${ }^{1,2}$, Rui Wang ${ }^{2}$, Bin Xian ${ }^{1,2}$, Chaoxiang Ren ${ }^{1,2}$, Qianqian Liu ${ }^{1,2}$, Qinghua Wu ${ }^{1,2}$ and Jin $\operatorname{Pei}^{1,2^{*}}$

\begin{abstract}
Background: Safflower (Carthamus tinctorius L.) is an important cash crop, of which the dried tube flower is not only an important raw material for dyes and cosmetics but also an important herb widely used in traditional Chinese medicine. The pigment and bioactive compounds are composed of flavonoids (mainly quinone chalcones), and studies have reported that MeJA can promote the biosynthesis of quinone chalcones, but the mechanism underlying the effect of MeJA in safflower remains unclear. Here, we attempt to use metabolomics and transcriptome technologies to analyse the molecular mechanism of flavonoid biosynthesis under MeJA treatment in safflower.

Results: Based on a UHPLC-ESI-MS/MS detection platform and a self-built database (including hydroxysafflor yellow A, HSYA), a total of 209 flavonoid metabolites were detected, and 35 metabolites were significantly different after treatment with MeJA. Among them, 24 metabolites were upregulated upon MeJA treatment, especially HSYA. Eleven metabolites were downregulated after MeJA treatment. Integrated metabolomics and transcriptome analysis showed that MeJA might upregulate the expression of upstream genes in the flavonoid biosynthesis pathway (such as CHSs, CHIs and HCTs) and downregulate the expression of downstream genes (such as F3Ms, ANRs and ANSs), thus promoting the biosynthesis of quinone chalcones, such as HSYA. The transcription expressions of these genes were validated by real-time PCR. In addition, the promoters of two genes $(\mathrm{CtCHI}$ and $\mathrm{CtHCT})$ that were significantly upregulated under MeJA treatment were cloned and analysed. 7 and 3 MeJA response elements were found in the promoters, respectively.

Conclusions: MeJA might upregulate the expression of the upstream genes in the flavonoid biosynthesis pathway and downregulate the expression of the downstream genes, thus promoting the biosynthesis of quinone chalcones. Our results provide insights and basic data for the molecular mechanism analysis of flavonoid synthesis in safflower under MeJA treatment.
\end{abstract}

Keywords: Flavonoid biosynthesis, MeJA treatment, Metabolomics, Transcriptome, Molecular mechanism, HSYA, Safflower

\footnotetext{
* Correspondence: peixjin@163.com

${ }^{1}$ State Key Laboratory of Characteristic Chinese Medicine Resources in Southwest China, Chengdu University of Traditional Chinese Medicine, Chengdu 611137, China

${ }^{2}$ College of Pharmacy, Chengdu University of Traditional Chinese Medicine,

Chengdu 611137, China
}

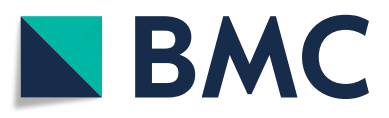

(c) The Author(s). 2020 Open Access This article is licensed under a Creative Commons Attribution 4.0 International License, which permits use, sharing, adaptation, distribution and reproduction in any medium or format, as long as you give appropriate credit to the original author(s) and the source, provide a link to the Creative Commons licence, and indicate if changes were made. The images or other third party material in this article are included in the article's Creative Commons licence, unless indicated otherwise in a credit line to the material. If material is not included in the article's Creative Commons licence and your intended use is not permitted by statutory regulation or exceeds the permitted use, you will need to obtain permission directly from the copyright holder. To view a copy of this licence, visit http://creativecommons.org/licenses/by/4.0/ The Creative Commons Public Domain Dedication waiver (http://creativecommons.org/publicdomain/zero/1.0/) applies to the data made available in this article, unless otherwise stated in a credit line to the data. 


\section{Background}

Safflower, Carthamus tinctorius L., is a member of the Asteraceae family and is an important economic plant worldwide. Its dried tubular flowers are an important raw material for dyes and cosmetics and are also an important herb widely used in traditional Chinese medicine. As a traditional Chinese medicine, the dried tubular flowers of safflower have been widely used to improve cerebral blood flow and to treat coronary heart disease, hypertension, and cerebrovascular diseases [1, 2]. Flavonoids, particularly quinone chalcones, are associated with these therapeutic effects. Among them, HSYA is the primary active component. It has the antioxidant activities and the myocardial and cerebral protective effects [3-5].

The flavonoid biosynthesis pathway is well understood, especially in some model plants, such as Arabidopsis [6, 7]. Chalcone synthase (CHS) catalyzes the reaction of one molecule of 4-coumaroyl-CoA and three molecules of malonyl-CoA into naringenin chalcone which is required for the flavonoids biosynthesis. It can be hydroxylated or glycosylated to the production of carthamone or HSYA. Chalcone isomerase (CHI) can convert naringenin chalcone into naringenin. In safflower, naringenin can be hydroxylated at the 6 position and glycosylated at the 6 and 7 positions to produce $5,6,7,4^{\prime}$-tetrahydroxyflavanone-6,7-diO- $\beta$-D-glucoside, or it can be hydroxylated at the 6 position and glycosylated at the 5 position to the production of 5,6 , 7,4' -tetrahydroxyflavanone-5-O- $\beta$-D-glucoside (neocartha$\mathrm{min})$. Additionally, flavanone 3-hydroxylase (F3H) can convert naringenin into dihydrokaempferol. It can be subsequently converted into kaempferol, and kaempferol can then be converted into quercetin [8]. With an emphasis on safflower, the research on flavonoid synthesis has gradually increased. To date, many flavonoid biosynthesis genes have been cloned in safflower, such as chalcone isomerase genes (CHIs) [9], chalcone synthase genes ( $\mathrm{CHSs}$ ) $[10,11]$, flavanone 3-hydroxylase genes (F3Hs) [12], UDPglucuronosyltransferases genes (UGTs) [13], and shikimate/ quinate hydroxycinnamoyltransferase genes (HCTs) [14].

As a well-known exogenous inducing factor, methyl jasmonate (MeJA) participates in many plant processes, ranging from plant defence to growth and development [15]. MeJA is of particular interest in plant cell engineering for producing bioactive compounds [16, 17]. It has been reported that flavonoids (mainly quinone chalcones) in safflower can be stimulated under MeJA treatment $[13,18]$. However, the molecular mechanism is largely unknown.

Recently, the application of metabolomics to medicinal plants has significantly facilitated the identification of the metabolic pathways of active medicinal compounds in plants. The UHPLC-ESI-MS/MS-based, widely targeted metabolomics method has become very popular in the field of analysis and identification of plant metabolites due to the advantages of high throughput, fast separation, high sensitivity, and wide coverage. Our methodology was based on a multiple reaction monitoring (MRM) approach [19], with a self-built compounds database according to the results of Chen et al. [20]. This approach has been widely applied in plant metabolite analysis in many plants, such as rice [20], tomato [21, 22], maize [23]. Furthermore, the integration of transcriptomics and metabolomics has larger advantages in revealing the biosynthetic mechanisms of key metabolic pathways [24-26]. Therefore, it is feasible to analyse the flavonoid biosynthesis pathway under MeJA treatment by the two technologies.

Here, metabolic profiling and differential flavonoid metabolites were screened based on a UHPLC-ESI-MS/ MS detection platform and a self-built database (including HSYA). In addition, transcriptome sequencing and differential transcripts were analysed. Integrated metabolomics and transcriptome sequencing was analysed based on the KEGG pathway, and the expression of different flavonoid biosynthesis genes with or without MeJA treatment were analysed by real-time PCR. The promoters of genes that were significantly upregulated under MeJA treatment were cloned and analysed. Our results provide insights and basic data for the regulation mechanism analysis of flavonoid synthesis under MeJA treatment.

\section{Results}

Metabolic profiling and differential flavonoid metabolite analysis

The flavonoid metabolites in safflower with and without MeJA treatment were investigated based on UHPLCESI-MS/MS and a self-built database (including HSYA). A total of 209 flavonoid metabolites were detected, including 62 flavones, 42 flavone C-glycosides, 40 flavonols, 20 flavanones, 18 anthocyanins, 11 isoflavones, 12 flavanols, 2 flavonolignans, 1 quinone chalcone, and 1 alkaloid. (Supplementary Table 1).

Orthogonal partial least squares-discriminant analysis (OPLS-DA) was used for variables with less correlation. As the experiment had biological duplication, the fold change and VIP value of the OPLS-DA model were combined to screen differential metabolites. There were 35 significantly different flavonoid metabolites between MeJA-treated and untreated materials. Among them, 24 metabolites were upregulated upon MeJA treatment, especially hydroxysafflor yellow A (HSYA). Eleven metabolites were downregulated after MeJA treatment. (Fig. 1a,b) The details of the different flavonoid metabolites were provided in Supplementary Table 2. The differential flavonoid metabolites from each comparison group were screened by using the KEGG database. The 


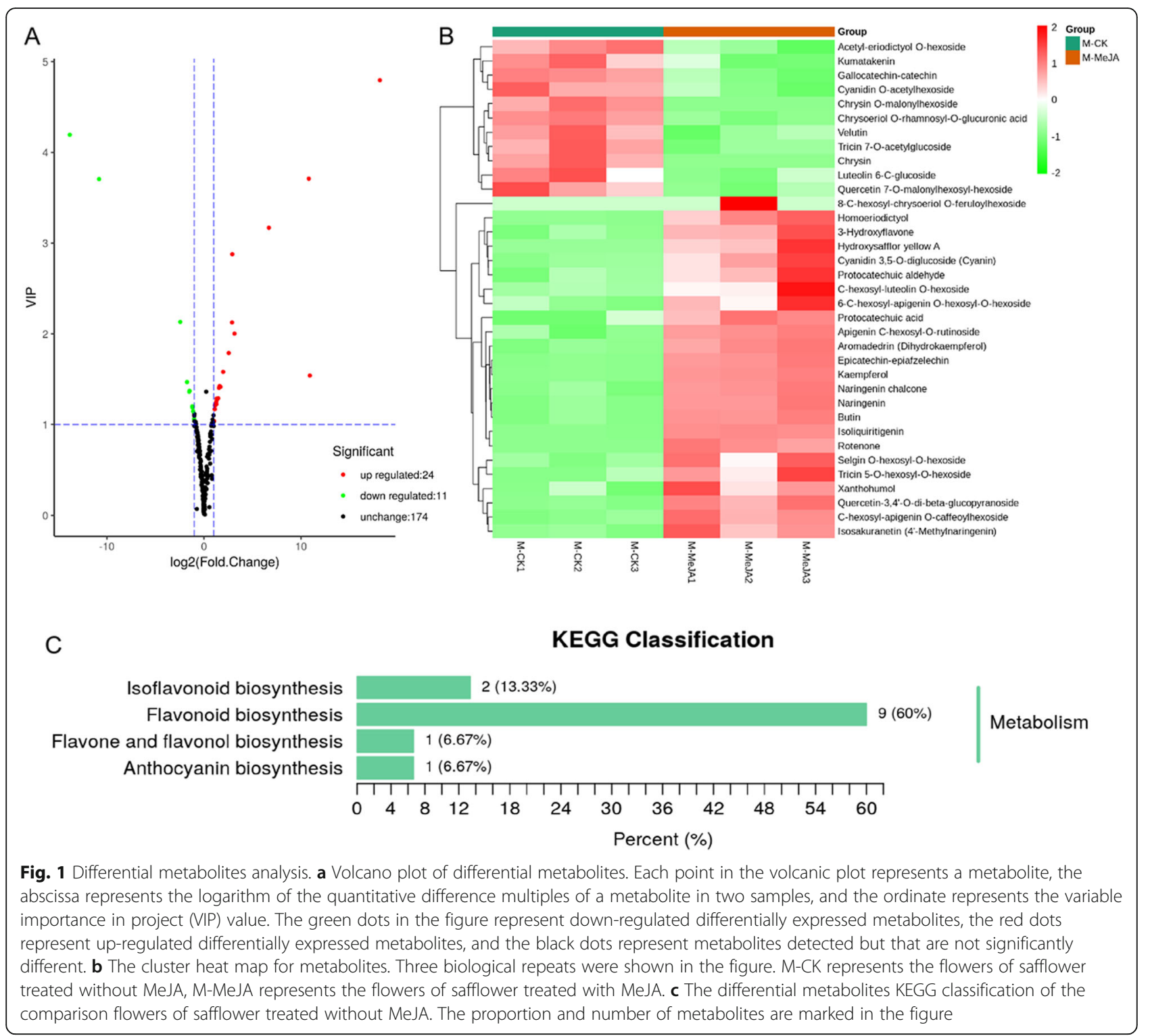

KEGG classification and enrichment analysis indicated that the differential flavonoid metabolites were mainly involved in the pathway of flavonoid biosynthesis (ko00941). (Fig. 1c).

\section{Transcriptome sequencing and differential transcript analysis}

The transcriptomes of the mixed safflower samples were sequenced. In the untreated samples, a $6.50 \mathrm{G}$ clean base was obtained $(44,185,480$ raw reads and 43,921,238 clean reads), and Q30 was 92.40\%. In the MeJA-treated samples, a $6.17 \mathrm{G}$ clean base was obtained $(42,087,290$ raw reads and 41,720,790 clean reads), and Q30 was 91.89\%. The different expressed genes were analysed with DESeq2. The total number of differentially expressed genes was 31,822 (20,741 upregulated genes and 11,081 downregulated genes). (Fig. 2).

The differential flavonoid metabolite transcripts from each comparison group were screened by using the KEGG database. The experiments focused on annotations in the phenylpropane metabolic pathway, including flavonoid biosynthesis (ko00941), anthocyanin biosynthesis (ko00942), isoflavonoid biosynthesis (ko00943), and flavone and flavonol biosynthesis (ko00944). The results showed that there were 61 significantly different flavonoid biosynthesis transcripts between MeJA-treated and untreated materials (43 in ko00941, 8 in ko00942, 5 in ko00943, and 5 ko00944). Most of transcripts were involved in flavonoid biosynthesis (ko00941). The details could be viewed in Supplementary Table S3. 


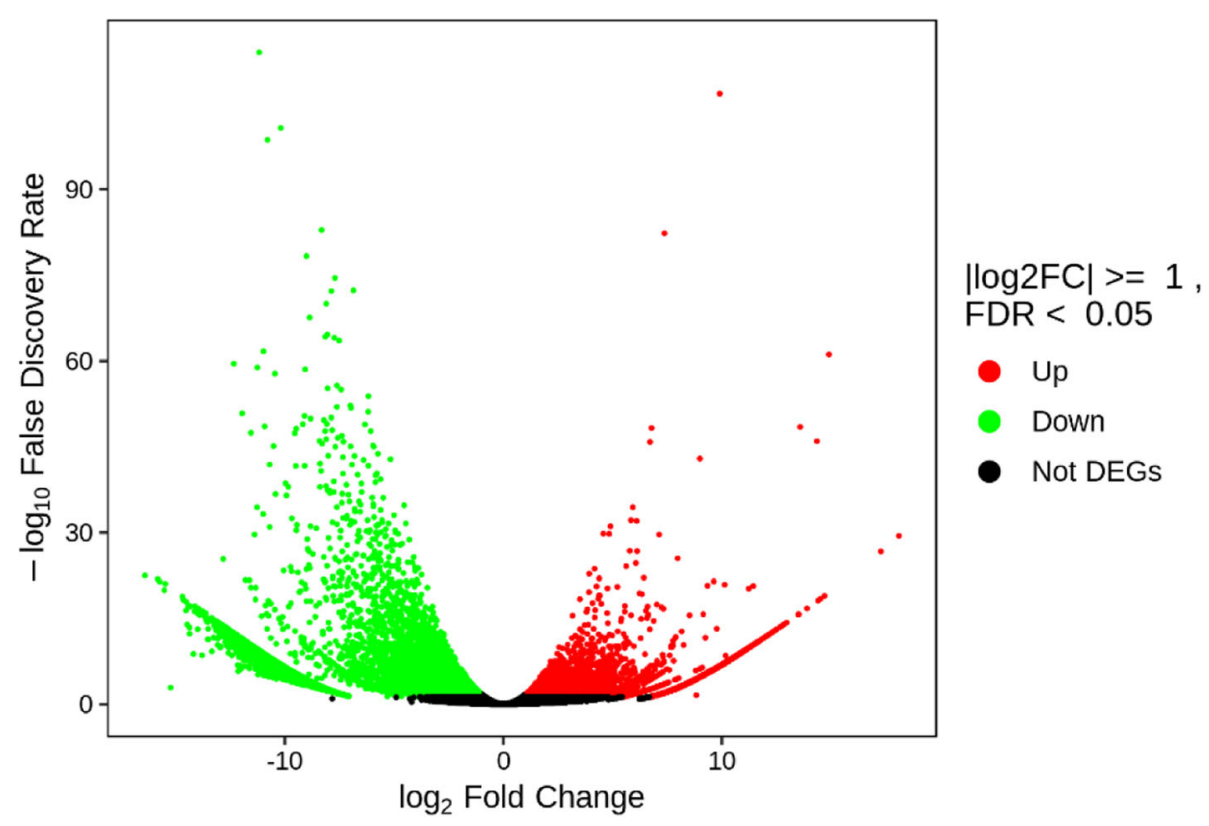

Fig. 2 Volcano plot of differential expression genes. The abscissa represents the change of gene expression multiple (log 2 Fold Change), and the ordinate represents the significant level of differentially expressed genes (-log10 False Discovery Rate). The expression of red gene was upregulated, that of green gene was down-regulated, and that of black gene was not significantly different

\section{Integrated analysis of the transcriptome and metabolome on the KEGG pathway}

The metabolic components were mapped onto the pathway of flavonoid metabolism (including ko00941, ko00942, ko00943 and ko00944) by combining metabolic components that had been detected by UHPLC-ESI-MS/MS, thus constructing the metabolic pathway map of integrative flavonoid biosynthesis in safflower. (Fig. 3) At the same time, the differentially annotated metabolites, together with the differentially annotated genes, were indicated on the integrated metabolic map. (Fig. 3) From the integrated metabolic map, it was shown that MeJA might upregulate the expression of the upstream genes in the flavonoid biosynthesis pathway (such as CHSs, CHIs, and HCTs) and might downregulate the expression of downstream genes (such as F3Ms, ANRs, and ANSs), thus promoting the biosynthesis of quinone chalcones, such as HSYA.

\section{Expression analysis of differentially expressed flavonoid biosynthesis genes by real-time PCR}

Real-time PCR was used to validate the transcriptome data. Ten differentially expressed genes were selected, including TRINITY_DN28401_c0_g1 (annotated as CHI), TRINITY_ DN36537_c1_g1 (annotated as HCT), TRINITY_DN37574_ c1_g2 (annotated as HCT), TRINITY_DN39063_c0_g1 (annotated as FLS), TRINITY_DN43344_c1_g1 (annotated as CHI), TRINITY_DN41734_c0_g5 (annotated as CHS), TRIN ITY_DN42700_c3_g2 (annotated as F3H), TRINITY_ DN43120_c5_g1 (annotated as ANR), TRINITY_DN44094_ c0_g1 (annotated as ANS), TRINITY_DN44565_c1_g1 (annotated as $F 3 M$ ). The primers used for this experiment could be found in Supplementary Table 4. Among them, HCT contains several transferase enzymes, which include anthranilate N-hydroxycinnamoyl/benzoyltransferase that catalyses the first committed reaction of phytoalexin biosynthesis. F3H can catalyse synthesis $\mathrm{N}$-terminal flavanone 3-hydroxylase. F3M can catalyse flavonoid, NADPH, $\mathrm{H}^{+}$, and $\mathrm{O}_{2}$ into 3 '-hydroxyflavonoid and $\mathrm{NADP}^{+}$, and $\mathrm{H}_{2} \mathrm{O}$, which functions like F3H. FLS catalyzes the formation of flavonols from dihydroflavonols. $\mathrm{CHS}$ catalyses the reaction of one molecule of 4-coumaroyl-CoA and three molecules of malonyl-CoA to form tetrahydroxychalcone. Chalcone isomerase converts tetrahydroxychalcone into naringenin. ANR and ANS are all involved in the synthesis of anthocyanins. The results showed that the expressions of 6 genes, including TRINITY_DN28401_c0_g1 (CHI), TRINITY_ DN36537_c1_g1 (HCT), TRINITY_DN37574_c1_g2 (HCT), TRINITY_DN39063_c0_g1 (FLS), TRINITY_DN43344_c1_ g1 (CHI) and TRINITY_DN41734_c0_g5 (CHS), were significantly upregulated, and 4 genes, including TRINITY DN42700_c3_g2 (F3H), TRINITY_DN43120_c5_g1 (ANR), TRINITY_DN44094_c0_g1 (ANS) and TRINITY_DN44565 c1_g1 (F3M), were significantly downregulated. (Fig. 4) From the integrated metabolic map, it could be seen that the expressions of upstream genes in the phenylpropane metabolic pathway were increased with MeJA treatment. And the results of gene expression also suggested that these genes might be involved in metabolic pathways. 


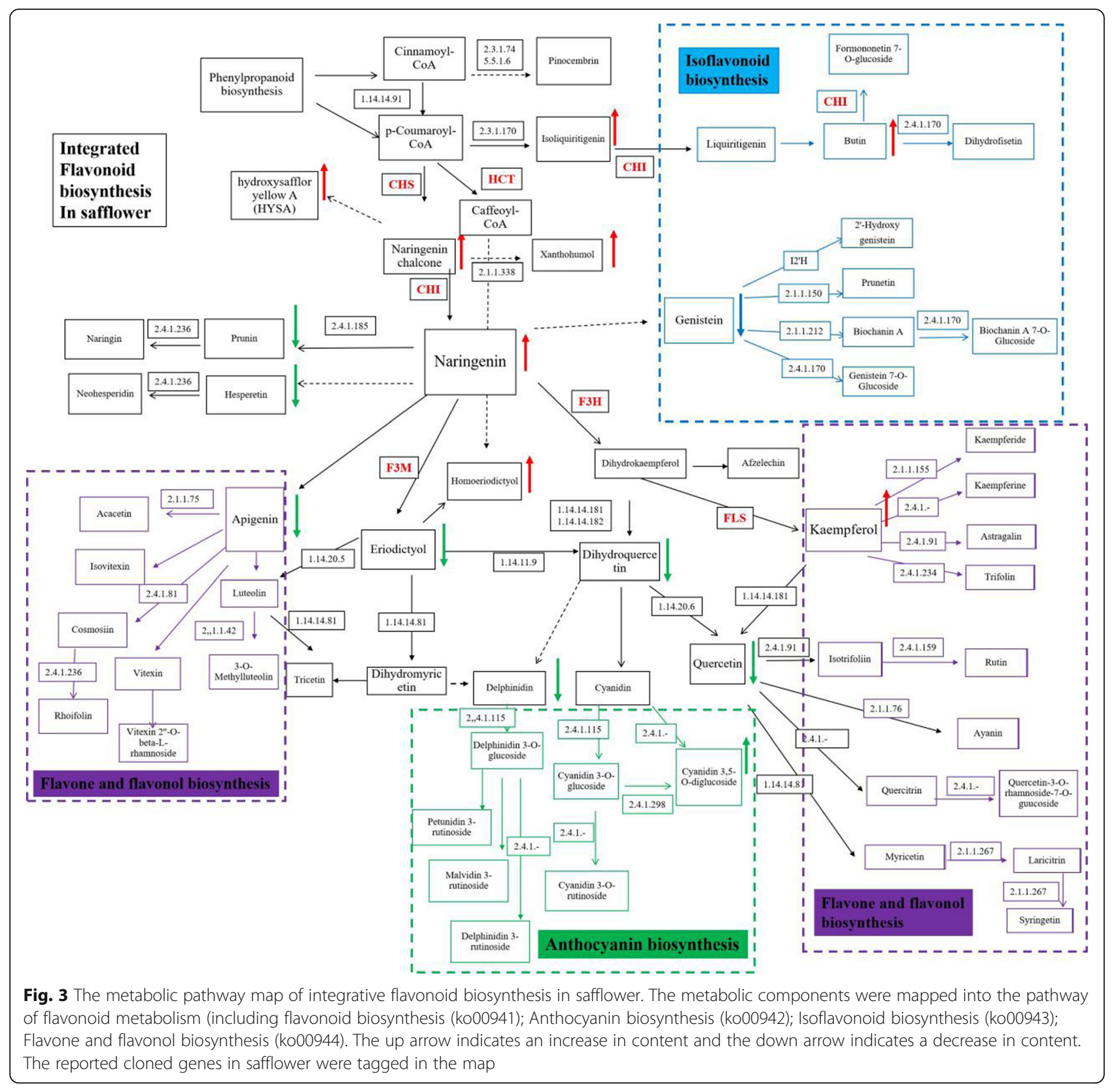

\section{Elements analysis of the promoters differentially expressed flavonoid biosynthesis genes}

To analyse how MeJA regulates gene expression, the promoters of differentially expressed flavonoid biosynthesis genes were cloned and analysed. Because there were no reference genome sequences from safflower, single oligonucleotide nested PCR (SON-PCR) was used to clone the promoters [27]. In our study, two promoters of MeJA significantly upregulated genes $\mathrm{CtCHI}$ and $C t H C T$ (named $p C t C H I$ and $p C t H C T$, respectively) were successfully cloned, and the fragments were cloned into the $\mathrm{T}$ vector and transformed into $\mathrm{DH} 5 \mathrm{a}$ bacteria. A positive bacterial solution was selected for sequencing. The sequences were shown in Fig. 5.

The sequences of the promoters were analysed using PlantCARE (http://bioinformatics.psb.ugent.be/webtools/ plantCARE) [28]. The sequences upstream of ATG were submitted to a web analysis program. Seven MeJA response elements could be found in the promoters of $p C t C H I$, while three MeJA response elements could be found in the promoters of $p C t H C T$. (Fig. 5) The results implied that MeJA could regulate these gene expressions through these elements, thus promoting the synthesis of quinone chalcones. 


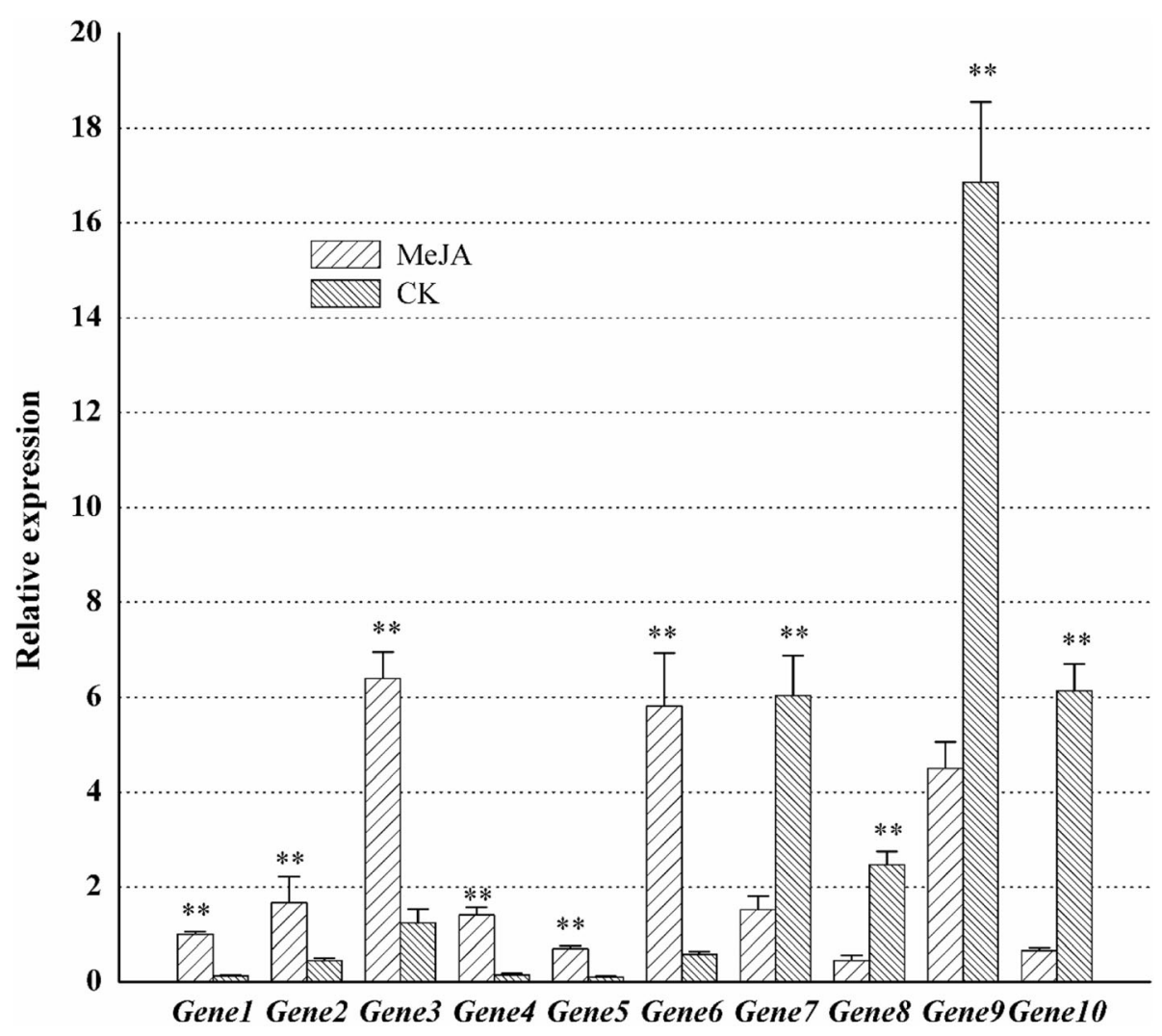

Fig. 4 Real-time PCR expression of 10 genes from the flavonoid biosynthesis pathway. 6 genes significantly upregulated and 4 genes significantly down-regulated are included. Gene1 represents TRINITY_DN28401_CO_g1 (annotated as CHI), Gene2 represents TRINITY_DN36537_C1_g1 (annotated as HCT), Gene3 represents TRINITY_DN37574_C1_g2 (annotated as HCT), Gene4 represents TRINITY_DN39063_CO_g1 (annotated as FLS), Gene5 represents TRINITY_DN43344_C1_91 (annotated as CHI), Gene6 represents TRINITY_DN41734_C0_g5 (annotated as CHS), Gene7 represents TRINITY_DN42700_C3_g2 (annotated as F3H), Gene8 represents TRINITY_DN43120_c5_gl (annotated as ANR), Gene9 represents TRIN ITY_DN44094_CO_gl (annotated as ANS), Gene10 represents TRINITY_DN44565_C1_gl (annotated as F3M)

\section{Discussion}

Many secondary metabolites can be induced by MeJA, such as volatile, stilbene, carotenoids, unsaturated fatty acids, flavonoids, lycopene, among others [29-31]. Flavonoids are one of the most reported compounds among them. However, not all the results showed that flavonoids biosynthesis can be upregulated by MeJA. Farag made the analysis of Erythrina lysistemon cell suspension culture in response to MeJA elicitation, and results revealed that triterpene i.e. oleanolic acid and fatty acid i.e. hydroxy-octadecadienoic acid were elicited in response to MeJA, whereas pterocarpans i.e. isoneorautenol showed a decline in response to MeJA elicitation [32]. Our results showed that 24 components of 35 differentially metabolized flavonoids could be induced and 11 components were reduced.

It have been reported that 104 compounds from safflower were isolated and detected [33]. In previous research [13], 12 flavonoid compounds were detected, including rutin, HSYA, kaempferol-3-O- $\beta$-D-rutinoside, kaempferol-3-O- $\beta$-D-glucoside, carthamin, luteolin, and
quercetin-3-O- $\beta$-D-glucoside. We found that rutin, HSYA, kaempferol-3-O- $\beta$-D-rutinoside, carthamin, and quercetin-3-O- $\beta$-D-glucoside were induced by MeJA treatment. In our results, 209 flavonoid compounds were detected by UHPLC-ESI-MS/MS with a self-built database. We profiled the chemical compositions of safflower in a systematic and comprehensive way, which provided a reference for their sufficient utilization in the future. UHPLC-ESI-MS/MS was an effective method to thoroughly understand plant secondary metabolites.

In previous reports of MeJA regulating flavonoids biosynthesis, many studies focused on the analysis of the genes expression. In Arabidopsis, MeJA similarly induced expression of almost all anthocyanin biosynthetic genes (PAL, CHS, CHI, F3H, F3'H, DFR and UFGT), but $P A L, C H S, C H I$ and $F 3 H$ were at only low levels. Further analysis show that the late anthocyanin biosynthesis genes (such as DFR and UFGT), were found to be upregulated strongly by MeJA [34]. While in Gynura bicolor DC., the expression of flavonoid biosynthesis genes, GbCHS, GbCHI, GbDFR and GbANS, was markedly up- 


\begin{abstract}
A
$\mathbf{2 1 0 8}$ CTGGTTAAGACTTGGTGTTGGGAAAAACCCACCCAAGTTTGAATCCGGGG GTGAGCAACCAGGAACCACTGAGTATTAATTCTAGGGGAGCATGCTTTAGCTCTTGGTAAAAATTACCAAGGATGTAGTCTGGACAAACCAAGATCAACCTTATAAAAAAAACT TCCTCAAAAAATAAATGAATTAGTTGGATCAAATATGTTACATAAACCAAAAAGAACATAAAATTGTGGGTCCATGGACACAGTTGCCAAGTCATACTTCCCTATCATCTTTCCTC TCTAAGTTTCAAATGACATTGCCAATTAACTTTTTTTCTAATGACTTTTTTGTTTAACTTTAATTTAATTATTCATATAGTACACTAAAACCAAATATCAATCAACCTCTTTTTTGTACA GATATGACG GTTTACTAACCTCTTACTGGATAAGTGTTTATCGATAAAAACATTACCTCACTTACCTTAATGTATAATGTTCTCGGAGAAAGGTTCAAATGAGAAACATAACTT TTTAAGAAAACGTGAGGAGCAATCTCAGCCACTCATCCGGTCAACAAAGGCGGTTTTGACCGTCGTCCCCCCTTTAATAGTCTCTACCCTCCCATGAATCGCGTCAATCCTT CGTGATCACGTCCACCGTAACCCAAGATGTGTGACTGAGATTGCTCCTCGTTTTTTTCTTAAATTGACATGCTTCTCGTATGAACCTCCCTCAATGTTCTCGACCATCAAAATTGATT GTTGATAATCATATTCAACCCGACAAACACTTACCGGATAAGAGTCTTTTCCCTCACTTTCTTGGTAAGAAGTTCGATCATTGTTATGCCCAGCAGTTTCCATACTCAATAGATAAG CACCGTAAAATAAATGAAACAGGAAAACACTTACCTGAATAAGTGTTTAGTAAAGGACGTCTTCTTATATAAGTTAATAATATTATTGAATAAGCTTTGAGCGCCTACTTGGTCTGA CCTTTGCATCATTTCCCATTTCCTCTATAACTATAAAAATAGCTACCAGCCTTCCTTCTGGTAGTTAAATTGGTCAACACCCCATTTACTCACCGGAAGTGCAATTACCATGGCTCC GCCGCCGTCCACCACCACCAGTCTTCAGGTCGAATCCGTCGTCTTTCCGCCATCCGTCAAGGCCCCGGGCACAGCCACCACTTTGTTCCTTGCAGGCGCAGGTACTCAAAT CTCAAAATCCGATCTTCAACTGACATTTAAGTTTCGATTAGGTGTACAGTTTTTTTTTTGTTTGAACGCTGGATAATTCACGCACATGTCCCGACACGAAGTCGACCAGGTATCCG GGATCCATTCGGGCACCCGACTAATCCTCGGCAACCATGGACCAACCCGAAAGCAGGGTATCGCGACATGAATCAAACCTGGATAGATAGGGATGCCATCCAGTGTCCCTCCCA AGGATGTTACCAAACCACTAGGCTACTAATGCAGGTGTAGGGTTTTTATTGATTTGAATCCTGTTTCTATAGGTGTGAGAGGTATGGAAATCGAAGGCAAGTTCGTCAAGTTA ACGGGGATTGGCCTGTATTTAGAGGATAAAGCCATTCCGTCACTCGCCGTTAAGTGGAAGGGCAAAACCGCGGCTGAGTTGATGGACTCCGTCCACTTCTACAGCGACATC ATTAATGGTAAT C GTCAACTTATTTTCGGAATCAAAAACTTATACAGATCGATCCCAAGGAATCGATGTCCTetTTTAGATGCAATTATTTAGGCGTTTTTTTTTGCATCGAACCA ACGAATGACTACCACGAGCACATACACCTCCCGGCGGTATAGCCGTATAGGGCAGCCACGGGCAGACCACTAATAAAAACACAAAATTCCCATCAAGCCTCATCTTTCCATCTAT TTTTAATTTTAGTAAAAACTCCAGGAGGAGCATATATTAACTTAACTTCTCACA TGACG ATACTTGGCCTATAAAAACATACATTTTCTTTTGAAGTACACCAGCTAAACTTTG GGTATATATTCTTCTTAAAACACAAACCGTGACTTACGTCTACATTCTCTTTCAGGCCCCTTTGAAAAACTTGCCGAGGTGGCAATGATAGTACCATTAGTCGGT -1
\end{abstract}

B $\mathbf{- 1 9 6 4}$ CAATtTTATTTCATTAGAACCCACTTTCATATCAGATGATTCAGATCTATTCCCACGTCAAATCCACTCCACCTTCTTCCTCCTCTCTTCCACCATCTCATTCC CCTGTCTCTTATACACATCTAGATGTGTATAAGAGACAGATCCTCACGTTCACAACCAAAAAGTGGTTTACATTTGAACTTTTCTCTCTCTATATATATATATATATAATCAAATGAAA ACTCATTTTATTGTGTAAACTTGAGGACTAATCTAGTCCATTAGATTTTAGTAATCAAGGGTTATTAAAACATGGAGAACACAATAACAATTCTTATGCTTTTTATTGGAACACACAT GATCATAAACTCATAGGGGCATTTTAGGCAATTTTATTTCATTAGAACCCACTTTCATATCAGATGATTCAGATCTATTCCCACGTCAAATCCACTCCACCTTCTTCCTCCTCTC TTCCACCATCTCATTCCCCTCCTCCACCGTCGCCGGCGCTCCACCACCGTCGGTGCTCCACCGTGTCTTACTCTCCTCCATCATCCACCATGAAAGACGACCTAGATCTCGAATTC GGTCCGGATCTGACCTCCAGACGCCTTCACGTTTCAAACTCCAGCCATAT TGACGCCTTCACCGCCGTTGCCACCACCACGACCGCCACCGCCTCCACCGCCACCACCACC ACCACCATCGTTCGTTCATACAAGATCTGGTAATTTGTATTTTCTCAGATTTCTCAATGAATGGCTCTTCATGTATCTATTGTGATGCACTCCAACTGTTCGACAAAATGTCTCCAAG AACAGTCTTTCATTCATTTGTTGTTACTCCTTTCAGATCTGGTGATGCGTTGCTCATATATGGTAATTTGCATTTTTAACTAAAAAACCCAATTTCTAGGGTTCTTGAGATTTTTTCC AAAATTAGGTTACTTTGATTCAATTTTTGAGATTGTTGTTTCACAGAAGTTGTTTGTAATCTTGATTTGTGTAACATATCTGAATTTTAACATTTTTTGTGGAGATTTTAAAAAATCA TTCTCGAAAAATTAGAGTTTTTTAAGTTTTGTTCATGAAGGTTTATCATGTGTTCTTGAAGGTTCATCATGTGATCTTTTGAAGGTTCATCATGTTCTTGAAGGCTGAAGTGGATGAT GATGTGAAGATTCATTAGATGAAAGCCGCCTTTATTAACCGAATGAGTGGCTGAGATTCGTCCTCACGTTCACAACCAAAAAGCTGTCTCTTATACACATCTAGATGTGTATAAGA GACAGCTTTTTGGTTGTGAACATGAGGGCCGCCGAGAGCGTGACACGTGGCGCTGTCTCTTATACACATCTAGATGTGTATAAGAGACAGCTTTTTGGTTGTGAACATGAGGGCC GCCGAGAGCGTGACACGTGGCGCTGTCTCTTATACACATCTAGATGTGTATAAGAGACAGCTTTTTGGTTGTGAACATGAGGACCGCCGAGAGCGTGACACGTGGCGTCTTTGA GTGTGGTCCCCTCCTTTAATTCAATGCATTCCTATGAATCGCGCCTAGTCATCCGCCGTGGAGCATATATATATATATATCCAACACATTATGCTTTTCTTTTTGTTAATTTTGCATCTG CTAATTCCATTGCTCCGTTGCTGCCTGCAGATAAACAATTACATCAATCATCATTATAATTGATTATCAACACGGTTAAGAATTTTGGAACAACCTCATTTGGTTCTTAATGTTCACA TCCAATATCGATATTTGTTCAAGAATGCCAAAATGCTTAAAAGTTCCATAAAATATATTAGCATTAATTTTTTAACCATGTGCATCAGTTGTGGCATGCACAAACAAAATGACAACA TTGGATCAATGAATGGATTTCGAAAGGGAAGGTCAATTAGCACCCAGCTTCACACTCTTTATAAAACAAGAGTTGTCGATGAAGCTGATCGATCATCATATCGTTCGAAA -1

Fig. 5 Identification of MeJA response elements found in the promoter sequences of $p C t C H I(\mathbf{a})$ and $p C t H C T$ (b). A is the sequence analysis for $p C t C H I . B$ is the sequence analysis for $p C t H C T$. The sequences were analyzed by PlantCARE (http://bioinformatics.psb.ugent.be/webtools/

plantCARE). The elements in the yellow indicated that the sequence of MeJA responded element was the same as it (sense strand of DNA), while the elements in the red was that as the reverse complementary sequence (antisense strand of DNA)

regulated. Compared with that in Arabidopsis, the genes, which are classified as up flavonoid biosynthesis genes, were found to be up-regulated strongly by MeJA [35]. From the studies above, it can be indicated that the flavonoid biosynthesis genes expression varied among different plants in response to the MeJA treatment. In our study, MeJA could upregulate the expression of upstream genes in the flavonoid biosynthesis pathway (CHSs, CHIs, and HCTs) and downregulate the expression of downstream genes (F3Ms, ANRs, and ANSs), thus promoting the biosynthesis of quinone chalcones, such as HSYA. It is probably that MeJA could upregulate the expression of TRINITY_DN28401_c0_g1 (CHI), TRINITY_ DN36537_c1_g1 (HCT), TRINITY_DN37574_c1_g2 (HCT), TRINITY_DN43344_c1_g1 (CHI), and TRINITY_DN41734 c0_g5 (CHS), and downregulate the expression of TRINITY DN42700_c3_g2 (F3H), TRINITY_DN43120_c5_g1 (ANR), TRINITY_DN44094_c0_g1 (ANS), and TRINITY_DN44565_ c1_g1 (F3M), thus promoting the biosynthesis of quinone chalcones (Fig. 4). Of course, to make more conclusive evidence, some other experiments, such as enzyme activity analysis and transgenic experiments, are required.
In the research of Gynura bicolor DC., some genes were markedly up-regulated under MeJA treatment, such as $G b C H S$, which is expressed more than 100-fold as compared with that before MJ treatment [35]. However, there was no such high change in our results. The main reason might be the different way of dealing with MeJA. In previous research, MeJA was added into $20 \mathrm{ml}$ fresh MS liquid medium, where Gynura bicolor DC. was planted, while in our research, MeJA was sprayed onto healthy safflower flowers. The amounts for plant exposed to MeJA were different.

Since there is no reference genome in safflower, it is still difficult to clone the fragment. However, we fortunately cloned two promoters of up-regulated gene (HCT and $C H I$ ) in our experiment. The sequence analysis results showed that there were MeJA response elements on the promoters, which further proved the RT-PCR results. Athough our results showed that MeJA upregulate the expression of upstream genes in the flavonoid biosynthesis pathway and downregulate the expression of downstream genes, thus promoting the biosynthesis of quinone chalcones, such as HSYA, what genes MeJA 
regulated to promote the production of HSYA is still unknown, as the genes involved in the biosynthesis of HSYA have not been fully identified. Future researches can be strengthened in this domain.

\section{Conclusions}

Here, we used metabolomics and transcriptome technologies to analyse the molecular mechanism of flavonoid biosynthesis under MeJA treatment in safflower. Based on a UHPLC-ESI-MS/MS detection platform and a self-built database (including HSYA), a total of 209 flavonoid metabolites were detected, and 35 metabolites were significantly different. Among them, 24 metabolites were upregulated upon MeJA treatment, especially HSYA. Eleven metabolites were downregulated after MeJA treatment. Integrated metabolomics and transcriptome analysis showed that MeJA might upregulate the expression of upstream genes in the flavonoid biosynthesis pathway (such as $\mathrm{CHSs}$, $\mathrm{CHIs}$ and $H C T s$ ) and downregulate the expression of downstream genes (such as F3Ms, ANRs and ANSs), thus promoting the biosynthesis of quinone chalcones, such as HSYA. The transcription expressions of these genes were validated by real-time PCR. In addition, the promoters of two genes that were significantly upregulated under MeJA treatment were cloned and analysed. Ten MeJA response elements were found in the promoters. Our results provide insights and basic data for the molecular mechanism analysis of flavonoid synthesis in safflower under MeJA treatment.

\section{Methods}

\section{Plant materials}

Safflower used in this experiment was named as "Chuanhonghua No.1", which is cultivated by Industrial Crop Research Institute, Sichuan Academy of Agricultural Sciences. It was presented by Renchuan Yao and identified as safflower (Carthamus tinctorius L.) by professor Pei Jin. It was cultivated at the medicinal botanical garden on the Wenjiang Campus of Chengdu University of Traditional Chinese Medicine. The treatment was primarily applied according to the previous report with some modifications [13]. A $100 \mu \mathrm{M}$ solution of MeJA (Sigma-Aldrich, Switzerland) was sprayed onto healthy safflower flowers 3 days after anthesis (DAA). In the control group, the flowers were sprayed with the same solution but without MeJA. The flowers were then enclosed in clear plastic bags to prevent the emission of volatile phytohormones and to allow for the elicit or solutions to be more highly absorbed. After treatment for $6 \mathrm{~h}$, the plastic bags were removed, and samples of flowers were collected, frozen immediately in liquid nitrogen and stored in a freezer at $-80^{\circ} \mathrm{C}$. For the RNA sequencing, five inflorescences of safflower were mixed as a sample. As the RNA sequencing results were verified by the real-time PCR experiments (the experimental method was listed below) in the study, there were no replicates for the RNA sequencing. For metabolism analysis, ten inflorescences of safflower plants were mixed as one sample. Three biological replicates were performed for the metabolomics analysis. The flowers of safflower with or without MeJA treatment are shown in Fig. 6.

\section{Sample preparation and extraction for metabolomic analysis}

The freeze-dried flowers were crushed using a mixer mill (MM 400, Retsch) with a zirconia bead for $1.5 \mathrm{~min}$ at 30 $\mathrm{Hz}$. One hundred milligrams of powder was weighed and extracted overnight at $4{ }^{\circ} \mathrm{C}$ with $1.0 \mathrm{~mL} 70 \%$ aqueous methanol. Following centrifugation at $10000 \mathrm{~g}$ for $10 \mathrm{~min}$, the extracts were absorbed (CNWBOND Carbon-GCB SPE Cartridge, $250 \mathrm{mg}, 3 \mathrm{~mL}$; ANPEL, Shanghai, China, www.anpel.com.cn/cnw) and filtered (SCAA-104, $0.22 \mu \mathrm{m}$ pore size; ANPEL, before LC-MS analysis.

\section{UHPLC conditions}

The sample extracts were analysed using an LC-ESI-MS/MS system (HPLC, Shim-pack UFLC SHIMADZU CBM30A system; MS, Applied Biosystems 6500 Q TRAP). The analytical conditions were as follows: UHPLC column, Waters ACQUITY UHPLC HSS T3 C18 $(1.8 \mu \mathrm{m}, 2.1 \mathrm{~mm} \times 100$ $\mathrm{mm})$; solvent system, A water $(0.04 \%$ acetic acid): B acetonitrile (0.04\% acetic acid); gradient program, $0 \% \mathrm{~B}$ at $0 \mathrm{~min}$, 95\% B at $11.0 \mathrm{~min}, 95 \% \mathrm{~B}$ at $12.0 \mathrm{~min}, 5 \% \mathrm{~B}$ at $12.1 \mathrm{~min}$, and $5 \% \mathrm{~B}$ at $15.0 \mathrm{~min}$; flow rate, $0.40 \mathrm{~mL} / \mathrm{min}$; temperature, $40^{\circ} \mathrm{C}$; and injection volume, $2 \mu \mathrm{L}$. The UHPLC effluent was connected to an ESI-triple quadrupole-linear ion trap $(\mathrm{Q}$ TRAP)-MS.

\section{ESI-q trap-MS/MS}

Mass spectrometry followed the method of Chen et al. [19]. LIT and triple quadrupole (QQQ) scans were acquired on a triple quadrupole-linear ion trap mass spectrometer (Q TRAP), API 6500 QTRAP LC/MS/MS system equipped with an ESI Turbo ion-spray interface, operating in positive ion mode and controlled by Analyst 1.6.3 software (AB Sciex, Waltham, MA, USA). The ESI source operation parameters were as follows: ion source, turbo spray; source temperature, $500^{\circ} \mathrm{C}$; ion spray voltage (IS), $5500 \mathrm{~V}$; the ion source gas I (GSI), gas II (GSII), and curtain gas (CUR) were set at 55,60, and $25.0 \mathrm{psi}$, respectively; and the collision gas (CAD) was high. Instrument tuning and mass calibration were performed with 10 and $100 \mu \mathrm{mol} / \mathrm{L}$ polypropylene glycol solutions in QQQ modes. QQQ scans were acquired as MRM experiments with collision gas (nitrogen) set to $5 \mathrm{psi}$. DP and CE for individual MRM transitions was performed with further DP and CE optimization. A specific set of 

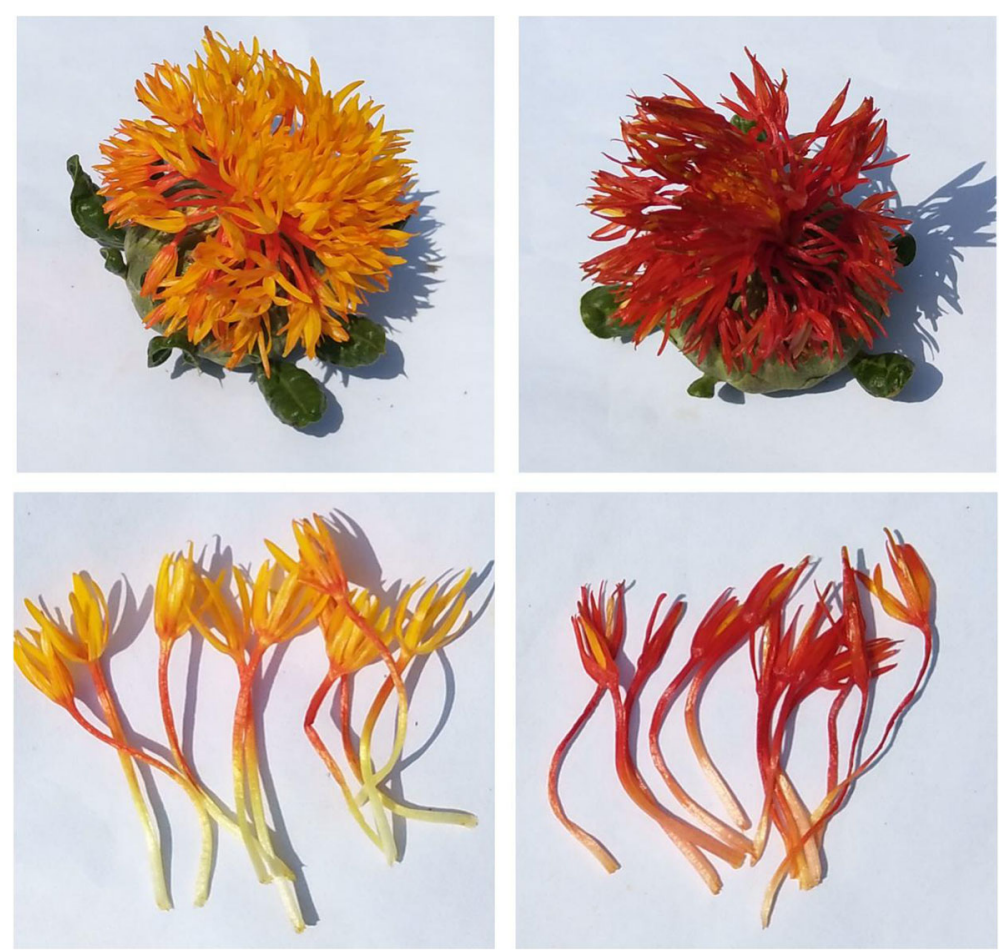

Fig. 6 The flowers of safflower treated with or without MeJA. a The inflorescences of safflower treated without MeJA (CK). b The inflorescences of safflower treated with MeJA. c The tubular flowers of safflower treated without MeJA (CK). D The tubular flowers of safflower treated with MeJA. The bar was $1 \mathrm{~cm}$

MRM transitions was monitored for each time period according to the metabolites eluted within that period.

\section{Qualitative and quantitative analysis of metabolites}

The flavonoid identification and quantification in our study was made according to a method of scheduled multiple reaction monitoring (MRM), which has been previously described [19]. With this methods, Chen made a genome-wide association analyses between genetic and biochemical, and reported 840 metabolites, from which 277 were identified or annotated [20]. Mainly based on this research, a database was built. All the metabolites in our study were detected or annotated by this database. The database and methods have been wildly used to detected or annotate the metabolites, such as the research of Wang [36] and Liu [37]. This methods is also called as wild-targetd analysis. As HSYA was not in the database, we use its standard sample (No.: MUST15072815, Chengdu Manster Biotechnology Co., LTD, China) to build a library and add it to the self-built database. The metabolites of the samples were qualitatively and quantitatively analysed by mass spectrometry. The characteristic ions of each substance were filtered by the triple quadrupole, and the signal strength of the characteristic ions was obtained in the detector. Chromatographic peaks were analyzed with Multi Quant software
3.0.3. The integrated area peak of each compound was used for the PCA and OPLS-DA analysis.

\section{Differential metabolite analysis}

Principal component analysis (PCA) can effectively extract main variance information and was used in many other research [29, 38]. In addition, orthogonal partial least squares-discriminant analysis (OPLS-DA) was used for variables with less correlation. The experiment had biological duplication; thus, the fold change and VIP value of the OPLS-DA model were combined to screen differential metabolites. PCA was analyzed by R software built-in functions (www.r-project.org/). The parameter: scale $=$ True. After conversion of the original data by $\log 2$, the data was centralized (Mean Centering) and analyzed by OPLSR. Anal of Metabo Analyst in R software. The main steps of our study can be referred to a previouly research [39]. Screening criteria: 1. Metabolites must exhibit fold changes $\geq 2$ and fold changes $\leq 0.5$. If a metabolite in the experimental groupwas more than 2 times or less than 0.5 times that of the metabolite in the control group, the difference was considered significant. 2. If there is biological duplication in the sample grouping, the metabolites of VIP $\geq 1$ are selected on the basis of the above. The VIP value indicates the influence intensity of the difference between groups of corresponding metabolites 
in the classification and discrimination of each group of samples in the model.

\section{RNA sequencing and annotation}

RNA isolation and purification and cDNA library construction and sequencing were performed as previously described [40]. All tissues were ground on dry ice, and total RNA was prepared by using TRIzol reagent (Invitrogen, CA, USA). To remove DNA, an aliquot of total RNA was treated with DNase (Takara, Dalian, China). RNA quantity and quality were determined by using a NanoDrop 2000 spectrophotometer (NanoDrop Technologies, Wilmington, DE, USA) and an Agilent 2100 Bioanalyser (Agilent Technologies, CA, USA), respectively. mRNA was isolated from total RNA using magnetic beads with oligo (dT) primer; cDNA was synthesized using a cDNA synthesis kit (TaKaRa, Dalian, China) and linking the sequencing adapter to both ends. The library preparations were sequenced on an Illumina HiSeq 4000 platform, and the unigene sequences obtained from our laboratory transcriptome database by RSEM (RNAseq by ExpectationMaximization) software were integrated for annotation. The whole set of transcript data can be found in the National Center for Biotechnology Information (NCBI) SRA database (SRR10011980 and SRR10011979).

\section{Screening of differential genes}

DESeq2 [41, 42] was used for differential expression analysis among sample groups. DESeq2 requires the input of unregulated read counting data of genes rather than RPKM (Reads Per Kilobase Million), FPKM (Fragments Per Kilobase Million) and other standardized data. Read counts of genes are the expected_count outputs calculated using RSEM (RNAseq by Expectation-Maximization). The expected count is generally lower than the read number. After discrepancy analysis, multiple hypothesis tests are needed to correct the hypothesis test probability ( $P$ value) with the Benjamini-Hochberg procedure to obtain the false discovery rate (FDR) when $\mid \log _{2}$ fold change $\mid \geq 1$ and $\mathrm{FDR}<0.05$.

\section{Real-time PCR analysis}

Real-time PCR analysis was performed as previously described [14]. Total RNA was isolated using an RNA extraction kit (Invitrogen, CA, USA), and reverse transcription was carried out using the prime script reagent kit (Takara, Dalian, China). The primers used to amplify the screened genes (CHS, CHI, HCT, etc.) by real-time PCR were designed by Primer 5.0, and parts of the safflower $28 \mathrm{~S}$ coding region were used as an internal reference gene. The primer details are listed in Supplementary Table 4. All of the primers were tested for their specificity by agarose gel electrophoresis. RT-PCR was performed using an SYBR prime script RT-PCR kit (Takara) with three replicates, and the cycling conditions were set according to the manual. The Bio-Rad CFX96 real-time PCR detection system (Hercules, CA, USA) was used in our experiment.

\section{Promoter cloning and sequence analysis}

Because there is no complete genome sequence for safflower, the $5^{\prime}$ flanking region of the flavonoid gene was isolated from genomic DNA of the safflower plant using SON-PCR. The reactions $(50 \mu \mathrm{L})$ were performed with $200 \mu \mathrm{mol}$ of each dNTP, $2 \mu \mathrm{mol}$ of primer and 2 units of LA Taq DNA polymerase (Takara). The primer details are listed in Supplementary Table 4. The resultant PCR fragment, measuring approximately $2 \mathrm{~kb}$, was cloned into a $p M D 19-T$ vector (Takara) and sequenced. The flavonoid gene promoter sequence was analysed with the PLACE Web SignalScan program (http://bioinformatics. psb. ugent.be/webtools/plantCARE [28];.

\section{Supplementary information}

Supplementary information accompanies this paper at https://doi.org/10. 1186/s12870-020-02554-6.

Additional file 1: Table S1. List of the 209 metabolites detected in safflower samples and OPLS-DA results. Two hundred nine flavonoid metabolites details were listed in the table. CK represent treatment without MeJA, M-MeJA represent treatment with MeJA. Three biological repeats were made in the experiment. Table S2. Extract of the 35 metabolites significantly different between MeJA and non-treated materials. CK represents treatment without MeJA. Three biological replicates were used in the experiment. Table S3. Details of the transcripts belonging to the flavonoid biosynthesis which were significantly different after MeJa treatment. M-CK represent treatment without MeJA, M-MeJA represent treatment with MeJA. Table S4. List of primers used for RT-PCR and promoter cloning experiments.

\section{Abbreviations}

$\mathrm{CHI}$ : Chalcone isomerase; CHS: Chalcone synthase; F3H: Flavonoid 3hydroxylase; UGT: UDP-glucuronosyl transferases; HCT: Hydroxylcinnamoyltransferase; F3Ms: Flavonoid 3'-monooxygenase;

ANRs: Anthocyanidin reductase; ANSs: Anthocyanidin synthase; MeJA: Methyl jasmonate; KEGG: Kyoto Encyclopedia of Genes and Genomes;

HSYA: Hydroxysafflor yellow A; CK: Control check; MeJA: Methyl jasmonate

\section{Acknowledgements}

We acknowledge Dr. Hu Zeng and Yuanyuan Qin (Metware Biotechnology Co., Ltd. Wuhan, China) for the data analysis, American Journal Experts (AJE) for the language editing (verification code: B631-386C-E812-C6A9-351B), and Renchuan Yao for providing us the plant materials.

\section{Authors' contributions}

JP conceived and designed the experiments. JC, JW and RW performed the experiments. QW and BX contributed to the material planting and sample collection. CR and QL contributed to data analysis. JC wrote the manuscript. All authors discussed the results and commented on the manuscript. The author(s) read and approved the final manuscript.

\section{Funding}

This project is supported by grants from the National Science Foundation of China $(81803669,81573544)$, which provide support for the test of metabolic and transcriptional, China Postdoctoral Science Foundation

(2018M643790XB), which provide support for the design and the writing of the study. 


\section{Availability of data and materials}

The raw data was uploaded to Sequence Read Archive (SRR10011980 and SRR10011979).

\section{Ethics approval and consent to participate}

This study has not directly involved humans, animals or plants.

\section{Consent for publication}

Not applicable.

\section{Competing interests}

The authors declare that they have no competing interests.

Received: 8 October 2019 Accepted: 19 July 2020

Published online: 29 July 2020

\section{References}

1. China T. S. P. C. o. Pharmacopoeia of the People's Republic of China, Part I. Beijing: Chemical Industry Press; 2015. p. 151.

2. Lou ZQ, Liu ML. A pharmacognostical study of the Chinese drug, Honghua,Carthamitinctor II. Acta Pharm Sin. 1956:4:233-9.

3. Qu C, Wang LY, Jin WT, Tang YP, Jin Y, Shi QX, Shang LL, Shang EX, Duan JA. Comparative analysis of the effects of Hydroxysafflor yellow a and Anhydrosafflor yellow B in safflower series of herb pairs using prep-HPLC and a selective knock-out approach. Molecules. 2016;21:1480-506.

4. Wei X, Liu H, Sun X, Fu FH, Zhang XM, Wang J, An J, Ding H. Hydroxysafflor yellow a protects rat brains against ischemia-reperfusion injury byantioxidant action. Neurosci Lett. 2005;386:58-62.

5. Zhu H, Wang Z, Ma C, Tian J, Fu F, Li C, Guo D, Roeder E, Liu K. Neuroprotective effects of hydroxysafflor yellow a: in vivo and in vitrostudies. Planta Med. 2003;69:429-33.

6. Winkel-Shirley B. Biosynthesis of flavonoids and effects of stress. Curr Opin Plant Bio. 2002;5:218-39.

7. Zhang Y, Butelli E, Martin C. Engineering anthocyanin biosynthesis in plants. Curr Opin Plant Bio. 2014;19:81-90.

8. Huang LL, Yang X, Sun P, Wen T, Hu SQ. The first illuminabased de novo transcriptome sequencing and analysis of safflower flowers. PLoS One. 2012; 7:e3865.

9. Ren CX, Tang XH, Chen CP, Chen J, Pei J, WU YY, Wu QH. Cloning and expression analysis of a new chalcone isomerase gene during flowering in safower. Turkish J Bot. 2019;43. https://doi.org/10.3906/bot-1809-25.

10. Shinozaki J, Kenmoku H, Nihei K, Masuda K, Noji M, Konno K, Asakawa Y, Kazuma K. Cloning and functional analysis of three chalcone synthases from the flowers of safflowers carthamus tinctorius. Nat Prod Commun. 2016; 11(6):787-90.

11. Guo D, Xue Y, Li D, He B, Jia X, Dong X, Guo M. Overexpression of CtCHS1 increases accumulation of Quinochalcone in safflower. Front Plant Sci. 2017; 8:1409-22.

12. Tu YH, Liu F, Guo DD, Fan LJ, Zhu ZX, Xue YR, Gao Y, Guo ML. Molecular characterization of flavanone 3-hydroxylase gene and flavonoid accumulation in two chemotyped safflower lines in response to methyl jasmonate stimulation. BMC Plant Biol. 2016;16:132-44.

13. Guo DD, Liu F, Tu YH, He BX, Gao Y, Guo ML. Expression patterns of three UGT genes in different chemotype safflower lines and under MeJA stimulus revealed their potential role in flavonoid biosynthesis. PLoS One. 2016;11: e0158159.

14. Chen J, Tang XH, Ren CX, Wei B, Wu YY, Wu QH, Pei J. Full-length transcriptome sequences and the identification of putative genes for flavonoid biosynthesis in safflower. BMC Genomics. 2018;19(1):548-60.

15. Wasternack C. Jasmonates: an update on biosynthesis, signal transduction and action in plant stress response, growth and development. Ann Bot. 2007;100:681-97.

16. Naoumkina MA, Farag MA, Sumner LW, Tang YH, Liu CJ, Dixon RA. Different mechanisms for phytoalexin induction by pathogen and wound signals in Medicago truncatula. P Natl Acad Sci USA. 2007:104:17909-15.

17. Suzuki H, Reddy MS, Naoumkina M, Aziz N, May GD, Huhman DV, Sumner LW, Blount JW, Mendes P, Dixon RA. Methyl jasmonate and yeast elicitor induce differential transcriptional and metabolic re-programming in cell suspension cultures of the model legume Medicago truncatula. Planta. 2005;220:696-707
18. He BX, Xue YR, Tu YH, Gao Y, Guo ML. CtCHS4 induces the accumulation of safflower quinone chalconesin response to methyl jasmonate induction. Acta Pharm Sin. 2018;53:636-54.

19. Chen W, Gong L, Guo Z, Wang W, Zhang H, Liu X, Yu S, Xiong L, Luo J. A novel integrated method for large-scale detection, identification, and quantification of widely targeted metabolites: application in the study of Rice metabolomics. Mol Plant. 2013;6:1769-80.

20. Chen W, Gao Y, Xie W, Gong L, Lu K, Wang W, Li Y, Liu X, Zhang H, Dong H, Zhang W, Zhang L, Yu S, Wang G, Lian X, Luo J. Genome-wide association analyses provide genetic and biochemical insights into natural variation in rice metabolism. Nat Genet. 2014:46:714-21.

21. Cho K, Cho KS, Sohn HB, Ha IJ, Hong SY, Lee H, Kim YM, Nam MH. Network analysis of the metabolome and transcriptome reveals novel regulation of potato pigmentation. J Exp Bot. 2016;67:1519-33.

22. Wen W, Li D, Li X, Gao Y, Li W, Li H, Liu J, Liu H, Chen W, Luo J, Yan JB. Metabolome-based genome-wide association study of maize kernel leads to novel biochemical insights. Nat Commun. 2014:5:3438-7.

23. Zhu G, Wang S, Huang Z, Zhang S, Liao Q, Zhang C, Lin T, Qin M, Peng M, Yang C, Cao X, Han X, Wang X, Knaap E, Zhang Z, Cui X, Klee H, Alisdair RF, Jie L, Huang S. Rewiring of the fruit Metabolome in tomato breeding. Cell. 2018:172:249-61.

24. Dong T, Han R, Yu J, Zhu M, Zhang Y, Gong Y, Li Z. Anthocyanins accumulation and molecular analysis of correlated genes by metabolome and transcriptome in green and purple asparaguses (Asparagus officinalis, L. ). Food Chem. 2019;271:18-28.

25. Li Y, Fang J, Qi X, Lin M, Zhong Y, Sun L, Cui W. Combined analysis of the fruit Metabolome and Transcriptome reveals candidate genes involved in flavonoid biosynthesis in Actinidia arguta. Int J Mol Sci. 2018;19(5):1471-87

26. Meng J, Wang B, He G, Wang Y, Tang X. WangS, Ma Y, Fu C, chai G, Zhou G. metabolomics integrated with Transcriptomics reveals redirection of the Phenylpropanoids metabolic flux in Ginkgo biloba. Agric Food Chem. 2019; 67(11):3284-91.

27. Antal Z, Rascle C, Fèvre M, Bruel C. Single oligonucleotide nested PCR: a rapid method for the isolation of genes and their flankingregions from expressed sequence tags. Curr Genet. 2004:46(4):240-6.

28. Lescot M, Déhais $P$, Thijs $G$, Marchal $K$, Moreau Y, Peer YVD, Rouzé $P$, Rombauts S. PlantCARE, a database of plant cis-acting regulatory elements and a portal to tools for in silico analysis of promoter sequences. Nucleic Acids Res. 2002;30(1):325-7.

29. Saccenti E, Hoefsloot HCJ, Smilde AK, Westerhuis JA, Hendriks MMW. Reflections on univariate and multivariate analysis of metabolomics data. Metabolomics. 2014:10:361-74.

30. Thakur M, Bhattacharya S, Khosla PK, Puri S. Improving production of plant secondary metabolites through biotic and abiotic elicitation. J Appl Res Med. 2018. https://doi.org/10.1016/j.jarmap.2018.11.004

31. Shi J, Ma CY, Qi DD, Lv HP, Yang T, Peng QH, Chen ZM, Lin Z. Erratum to: transcriptional responses and flavor volatiles biosynthesis in methyl jasmonate-treated tea leaves. BMC Plant Biol. 2017;136. https://doi.org/10. 1186/s12870-017-1076-5

32. Farag MA, Mekky H, El-Masry S. Metabolomics driven analysis of Erythrina lysistemon cell suspension culture in response to methyl jasmonate elicitation. J Adv Res. 2016:7(5):681-9.

33. Zhou X, Tang $L, X u Y$, Zhou G, Wang Z. Towards a better understanding of medicinal uses of Carthamustinctorius $L$. in traditional Chinese medicine: a phytochemical and pharmacological review. J Ethnopharmacol. 2014;151(1): 27-43.

34. Shan XY, Zhang YS, Peng W, Wang ZL, Xie DX. Molecular mechanism for jasmonate-induction of anthocyanin accumulation in Arabidopsis. J Exp Bot. 2009:60(13):3849-60.

35. Shimizu Y, Maeda K, Kato M, Shimomura K. Co-expression of GbMYB1 and GbMYC1 induces anthocyanin accumulation in roots of cultured Gynura bicolor DC. Plantlet on methyl jasmonate treatment. Plant Physiol Bioch. 2011:49(2):159-67.

36. Wang A, Li R, Ren L, Gao X, Zhang Y, Ma Z, Ma D, Luo Y. A comparative metabolomics study of flavonoids in sweet potato with different flesh colors (Ipomoea batatas (L.) lam). Food Chem. 2018;260:124-34

37. Liu YH, Lv JH, Liu ZH, Wang J, Yang BZ, Chen WC, Ou LJ, Dai XZ, Zhuqing Z, Xuexiao Z. Integrative analysis of metabolome and transcriptome reveals the mechanism of color formation in pepper fruit (Capsicum annuum L.). Food Chem. 2020:306. https://doi.org/10.1016/j.foodchem.2019.125629. 
38. Li Q, Song J. Analysis of widely targeted metabolites of the euhalophyte Suaeda salsa under saline conditions provides new insights into salt tolerance and nutritional value in halophytic species. BMC Plant Biol. 2019; 19:388-98.

39. Ying H, Shi J, Zhang S, Pingcuo G, Wang S, Zhao F, Cui YN, Zeng XL. Transcriptomic and metabolomic profiling provide novel insights into fruit development and flesh coloration in Prunus mira Koehne, a special wild peach species. BMC Plant Biol. 2019;19:463-78.

40. Wang Z, Cui Y, Vainstein A, Chen S, Ma H. Regulation of fig (Ficuscarica L.) fruit color: Metabolomic and Transcriptomic analyses of the flavonoid biosynthetic pathway. Front Plant Sci. 2017;8:1990-2004.

41. Love Ml, Huber W, Anders S. Moderated estimation of fold change and dispersion for RNA-Seq data with DESeq2. Genome Bio. 2014;15:550-70.

42. Varet H, Loraine BG, Coppée JY, Dillies MA. SARTools: a DESeq2- and EdgeRbased R pipeline for comprehensive differential analysis of RNA-Seq data. PLoS One. 2016;11(6):e0157022.

\section{Publisher's Note}

Springer Nature remains neutral with regard to jurisdictional claims in published maps and institutional affiliations.

Ready to submit your research? Choose BMC and benefit from:

- fast, convenient online submission

- thorough peer review by experienced researchers in your field

- rapid publication on acceptance

- support for research data, including large and complex data types

- gold Open Access which fosters wider collaboration and increased citations

- maximum visibility for your research: over $100 \mathrm{M}$ website views per year

At BMC, research is always in progress.

Learn more biomedcentral.com/submissions 